\title{
A Three-Dimensional Analysis of Marine Radar Images for the Determination of Ocean Wave Directionality and Surface Currents
}

\author{
I. R. Young AND W. RosenthaL \\ Institut für Meereskunde der Universität Hamburg and Max-Planck-Institut für Meteorologie, Hamburg, Federal Republic of Germany \\ F. ZIEMER \\ Institut für Meereskunde der Universität Hamburg and Deutsches Hydrographisches Institut, Hamburg, Federal Republic of Germany
}

A series of spatial wave images recorded by a conventional marine radar is analyzed to determine the three-dimensional $E\left(k_{x}, k_{y}, \omega\right)$ spectrum. In the absence of a surface current the spectral energy in this three-dimensional wave number frequency space will lie on a shell defined by the dispersion relationship. Any deviation from the expected dispersion relationship can be interpreted as being due to a current induced Doppler shift of the wave frequency. A least squares curve fitting technique is used to determine the surface current required to account for the observed Doppler shift. A comparison of the radardetermined spectra and surface currents with ground truth data indicates that the radar system and analysis technique produces results consistent with conventional instrumentation.

\section{INTRODUCTION}

In recent years, considerable interest has been shown in the application of remote sensing techniques to the measurement of ocean waves and currents. These studies have largely dealt with the use of three forms of radar systems: SAR (synthetic aperture radar) [Alpers, 1983], SLAR (side-looking aperture radar) [McLeish and Ross, 1983], and HF (high-frequency) radar [Barrick et al., 1977]. Because of the size of the antennas required, HF radar has only been deployed as a land-based system. SAR and SLAR systems, however, have been successfully used in aircraft and orbiting satellites, thus allowing large areas of the oceans to be scanned.

In addition to these widely reported remote sensing techniques, some limited interest has also been shown in the use of conventional marine imaging radar for the collection of ocean data. Ijima et al. [1964] and Wright [1965] were among the first to report the use of such radar for imaging ocean waves. Oudshoorn [1960], Willis and Beaumont [1971], Evmenov et al. [1973], and Mattie and Harris [1979] have also reported wave images obtained by radar. These researchers visually inspected the radar images to obtain estimates of the mean wave direction, wave length, and period.

Hoogeboom and Rosenthal [1982] and Ziemer et al. [1983] have extended these early findings by digitizing the radar images and finding their two-dimensional Fourier transforms. They have shown that the two-dimensional spectrum of the digitized radar image is similar to the spectrum obtained from conventional buoy data. In addition, the spectral value at each wave number appears to be a well defined function of the corresponding value of the directional wave spectrum. Hence it appears that such radar systems can be successfully used to determine the directional properties of waves and perhaps even the full directional spectrum.

A major problem exists in the interpretation of twodimensional wave number spectra, whether they be obtained

Copyright 1985 by the American Geophysical Union.

Paper number $4 \mathrm{C} 1006$.

0148-0227/85/004C-1006\$05.00 from ship radar or any other imaging system, since a $180^{\circ}$ directional ambiguity exists in the resulting spectra. For relatively simple wave spectra this ambiguity can be removed by assuming that the mean wave direction will be closely related to the wind direction. For more complex sea conditions, where there may be waves propagating at large angles to the wind, or if the wind direction is unknown, such a method is no longer applicable.

Atanassov et al. [this issue] have shown that the directional ambiguity can be removed by the use of two images, separated by a short time interval, together with knowledge of the dispersion relationship for gravity waves. In this study an analysis technique is developed for a full time series of radar images; each taken at a successive revolution of the radar antenna. By using this series of images, the three-dimensional energy density spectrum $E\left(k_{x}, k_{y}, \omega\right)$ can be found.

Since, in the limit of linear theory, surface waves exhibit a uniquely defined dispersion relationship between $|\mathbf{k}|$ and $\omega$, the energy density in three-dimensional wave number frequency space has nonvanishing values only on the two-dimensional shell defined by this dispersion relationship. As an application of the analysis technique, experimental data is analyzed to determine the oceanic current that causes a Doppler shift in the dispersion relationship for the surface waves.

\section{Radar Imagng of Waves}

The fact that marine radars give returns from ocean waves has been known for many years. Indeed, such returns (sea clutter) can pose a serious problem in marine navigation [Watson-Watt, 1978], as they can obscure echoes from small objects such as buoys. Indeed, special sea clutter controls are fitted to most commercial radar systems to facilitate the suppression of the returns from waves. Figure 1 shows a photograph of the radar screen (plan position indicator or PPI) in which wave images are clearly visible.

The interaction of electromagnetic waves and water waves has been extensively considered by numerous authors, and no attempt will be made here to review this work in any detail. Although the processes which cause a radar return from water waves are still not fully understood, it appears that a return 


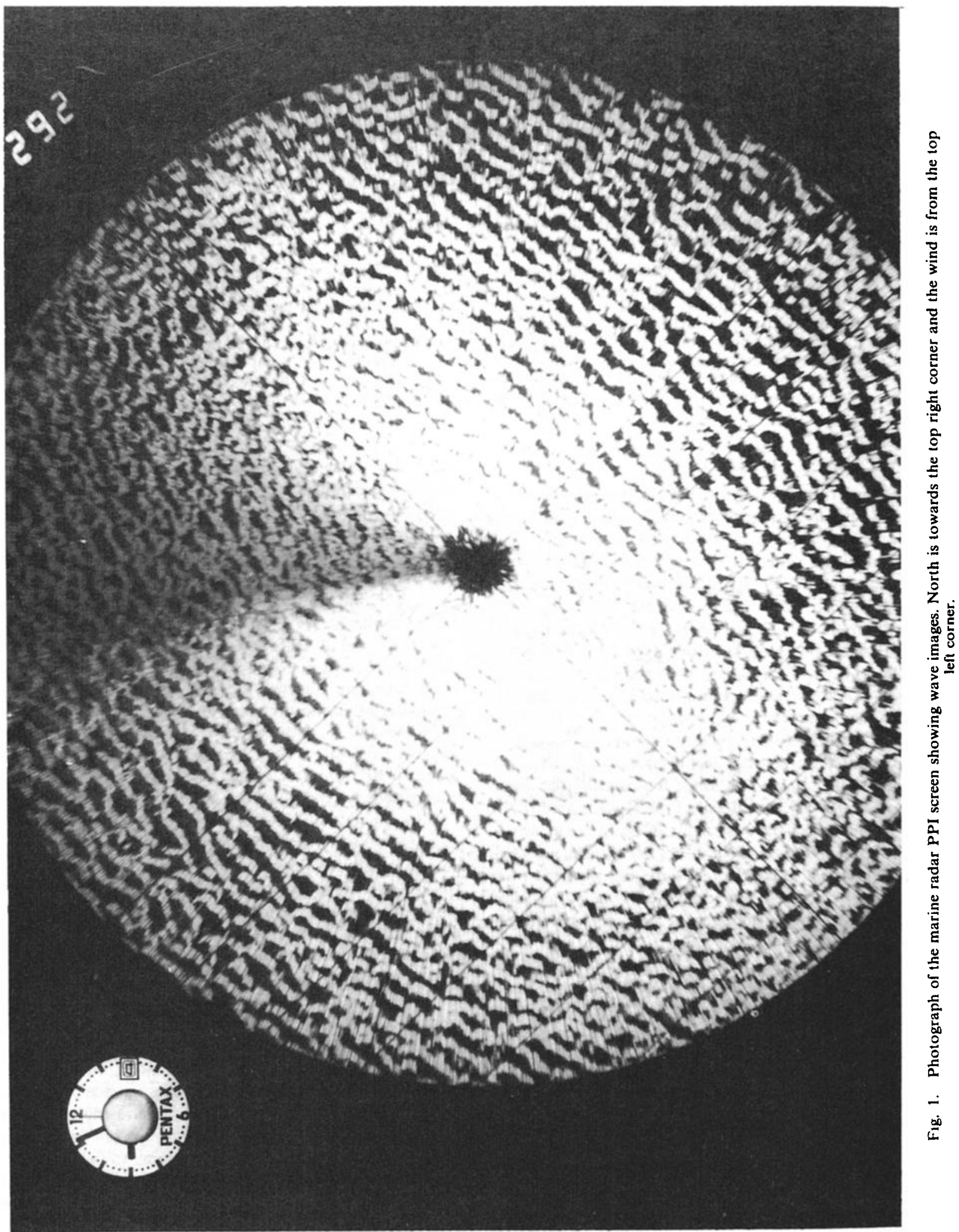


can be caused by either specular reflection (mirrorlike reflection) or by Bragg-resonant diffraction backscatter.

Specular reflection is important at small angles of incidence (angle between radar wave and the normal to the water surface) [Valenzuela, 1978]. Thus radar altimeters and other radars that look straight down on the sea obtain returns from such a mechanism. At larger angles of incidence, Bragg scattering is responsible for the return, at the grazing incidence Bragg scattering criterion being fulfilled by those waves having a wavelength equal to one half the electromagnetic wavelength. At present, commercially available marine radars use as their smallest electromagnetic wavelength approximately $3 \mathrm{~cm}$ ( $X$ band). Hence it is the high-frequency gravity capillary waves which cause a radar return. The longer waves which are of interest can be imaged by the radar, since they modulate the short Bragg scattering waves [Valenzuela, 1978]. Valenzuela has also indicated that toward grazing incidence, which is the case for ship mounted radar, other more complex mechanisms may become significant. These mechanisms include shadowing [Wagner, 1967; Smith, 1967; Seltzer, 1972], diffraction [Kolmykov and Pusovoytenko, 1976], and trapping by atmospheric ducts and surface waves [Brekhovskikh, 1960]. Although these uncertainties as to the mechanisms which cause imaging of ocean waves by marine radar place limitations on determining the transfer function between the surface wave spectrum and the radar spectrum, other properties such as wave direction, frequency, and phase speed can still be obtained.

\section{Multidimensional Fourier Analysis}

Consider the wave level elevation $\eta(\xi)$, where $\xi$ is the threedimensional space-time vector $(x, y, t), x$ and $y$ are the horizontal spatial coordinates, and $t$ is time. The multidimensional Fourier series of $\eta(\xi)$ is [Bath, 1974]

$$
F(\Omega)=\sum_{j=0}^{N-1} \eta\left(\xi_{j}\right) \exp \left(-i \Omega \cdot \xi_{j}\right)
$$

where $\eta\left(\xi_{j}\right)$ is the water level elevation corresponding to the space-time vector $\xi_{j}, \Omega$ is the wave number frequency vector, and the vector product $\Omega \cdot \xi$ is defined as $\Omega \cdot \xi=k_{x} x+k_{y} y$ $-\omega t$. Noting that $\eta(\xi)$ is a real-valued function, the complex conjugate of $(1)$ is

$$
F^{*}(\Omega)=\sum_{j=0}^{N-1} \eta\left(\xi_{j}\right) \exp \left(i \Omega \cdot \xi_{j}\right)
$$

From (1) and (2),

$$
F(\boldsymbol{\Omega})=F^{*}(-\boldsymbol{\Omega})
$$

The variance spectrum can be defined in terms of the Fourier transform as

$$
E(\Omega)=\frac{1}{L_{x} L_{y} T}|F(\Omega)|^{2}
$$

where $L_{x}$ and $L_{y}$ are the lengths of the space series in the $x$ and $y$ directions respectively and $T$ is the length of the time series. The normalization factor in (4), $1 / L_{x} L_{y} T$, is chosen such that

$$
\int_{-\mathbf{\Omega}_{N}}^{\mathbf{\Omega}_{N}} E(\boldsymbol{\Omega}) d \mathbf{\Omega}=\sigma^{2}
$$

where $\Omega_{N}$ is the Nyquist limit and $\sigma^{2}$ is the variance of the wave record. Equations (3) and (4) give

$$
E(\boldsymbol{\Omega})=E(-\boldsymbol{\Omega})
$$

and hence the spectrum is symmetric to reflections about $\boldsymbol{\Omega}=\mathbf{0}$. If a two-dimensional analysis is considered, (6) becomes $E\left(k_{x}, k_{y}\right)=E\left(-k_{x},-k_{y}\right)$ and, unless other information is used, there is no way of determining in which direction waves are propagating. For a three-dimensional analysis, however (6) yields $E\left(k_{x}, k_{y}, \omega\right)=E\left(-k_{x},-k_{y,}-\omega\right)$.

For each $\mathbf{\Omega}$ coordinate the phase speed direction of the corresponding plane wave in $x, y, t$ space is given by the time development of a fixed phase plane:

$$
\Omega \cdot \xi=\left(k_{x} x+k_{y} y-\omega t\right)=\text { const }
$$

Consequently, two points in $\boldsymbol{\Omega}$ space lying mirror symmetric about the $k_{x}, k_{y}$ plane will differ in phase speed direction by $180^{\circ}$; the phase speed direction jumps when the $k_{x}, k_{y}$ plane is crossed.

The two-dimensional spectrum $E\left(k_{x}, k_{y}\right)$ can be obtained by integrating $E(\Omega)$ with respect to the $\omega$ coordinate and thereby losing the information concerning the phase speed direction, since (6) will lead to a symmetric $E\left(k_{x}, k_{y}\right)$. However, if only one half plane, either $\omega>0$ or $\omega<0$, is used in the integration, the resulting two-dimensional spectrum

$$
\tilde{E}\left(k_{x}, k_{y}\right)=\int_{\omega>0} E(\boldsymbol{\Omega}) d \omega
$$

is, in general, asymmetric and the direction of $\mathbf{k}=\left(k_{x}, k_{y}\right)$ is the phase speed direction. If the negative half plane of frequency space is used in the integration, $\mathbf{k}$ indicates the direction opposite to the phase speed direction.

\section{The Influence of Surface Currents}

As a first-order approximation it can be assumed that gravity waves will follow the dispersion relationship

$$
\omega_{0}^{2}=g|\mathbf{k}| \tanh (|\mathbf{k}| d)
$$

where $\mathbf{k}$ is the wave number vector, $d$ the water depth, and $g$ the gravitational acceleration. Should there be a current $\tilde{U}$ relative to the observer, it will cause a Doppler shift in the wave frequency

$$
\omega=\omega_{0}+\boldsymbol{k} \cdot \tilde{\mathbf{U}}
$$

where $\omega$ is called the frequency of encounter. In threedimensional wave number frequency space, (9) represents a shell which is invariant to rotations about the frequency axis. For a given value of frequency the dispersion relationship represents a circle with radius $|\mathbf{k}|$ and origin at $k_{x}=k_{y}=0$. In the presence of a current, however, this symmetric structure is distorted. Figure 2 shows a plot of (10) for various values of $\tilde{U}$; the dispersion relationship is now taking on an elongated shape as it is a function of $|\tilde{U}|$ and the angle to the current.

Stewart and Joy [1974] have shown that rather than being the surface current, the component of $\tilde{U}$ in the direction of the wave number vector $\mathbf{k}$ is a weighted mean current over the upper layer of the ocean. Extending their result to consider the full current vector yields

$$
\overline{\mathbf{U}}=2|\mathbf{k}| \int_{-d}^{0} \mathrm{U}(z) \exp (2|\mathbf{k}| z) d z
$$

In general, $\mathbf{U}(z)$ is the superposition of the tidal current $\mathbf{U}^{t}$, other currents associated with ocean circulations $U^{\text {os }}$, the wind drift $\mathbf{U}^{d}$, wave-induced currents $\mathbf{U}^{w}$, and ship motion $\mathbf{U}^{\text {sm }}$ [Alpers et al., 1981]:

$$
\mathbf{U}(z)=\mathbf{U}^{t}+\mathbf{U}^{\mathrm{os}}+\mathbf{U}^{d}+\mathbf{U}^{w}+\mathbf{U}^{\mathrm{sm}}
$$

The wind drift current $\mathbf{U}^{d}$ and the mean ocean circulation $\mathbf{U}^{\text {os }}$ cannot always be separated, but here it is assumed that $\mathbf{U}^{d}$ is 


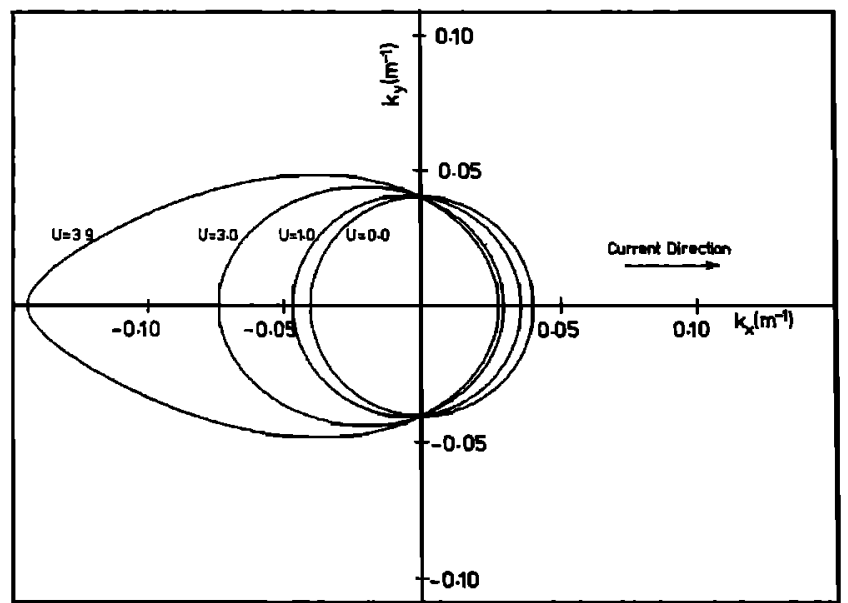

Fig. 2. The dependence of the dispersion relationship, in wave number space, on the angle to the current vector. The wave frequency is $0.1 \mathrm{~Hz}$, and the water depth is infinite. The current of $3.9 \mathrm{~m} \mathrm{~s}^{-1}$ represents the kinematical limit. For velocities greater than this, wave energy can no longer be propagated against the stream.

the current in the upper layer of the ocean driven by the wind stress (the Ekman layer) whereas $\mathbf{U}^{\text {os }}$ is the deeper current driven by the density field. The wave-induced current $U^{w}$ includes the effects of the Stokes drift [Phillips, 1977] as well as the influence of nonlinear wave-wave interactions [LonguetHiggins and Phillips, 1962].

\section{Radar System and data Processing}

The radar used in this study was an $X$ band Raytheon (Ray TM/EP1650/9xR) navigation system. Detailed system parameters are shown in Table 1 . The radar is of the conventional rotating beam type, which uses a plan position indicator (PPI) to display the image.

The system for recording and storing the images is shown schematically in Figure 3. The radar images as displayed on the PPI were recorded on photographic film using a 35-mm camera with a motor drive and a shutter synchronized with the radar sweep. During the full radar antenna sweep of approximately $2.2 \mathrm{~s}$ the shutter was kept open. Upon completion of the sweep the shutter was closed and the film advanced to the next frame. The shutter was then reopened immediately, and the cycle repeated. Thus the radar image at each successive rotation of the antenna was recorded. Since the time taken to advance the film was finite, there was a small section of the radar sweep on each image where the camera shutter was closed. This region appears as the shadowed sector in Figure 1. Care was taken in later analysis to avoid the use of this portion of the image.

The photographic images were digitised using an Optronics Colormation System C 4500 image processor. The digitized image consisted of $512 \times 512$ pixels each representing the grey scale intensity at one of a possible 256 levels. The radar

TABLE 1. Properties of the $X$ Band Raytheon Navigation Radar

\begin{tabular}{ll}
\hline \multicolumn{1}{c}{ Property } & \multicolumn{1}{c}{ Value } \\
\hline Frequency & $9.4 \mathrm{GHz}$ \\
Pulse repetition frequency & $3600 \mathrm{~Hz}$ \\
Antenna type & $2.75-\mathrm{m}$ slotted wave guide \\
Antenna beam width at $3 \mathrm{~dB}$ & $0.85^{\circ}$ horizontal, $22^{\circ}$ vertical \\
Polarization & $\mathrm{HH}$ \\
Rotation speed & $27 \mathrm{rpm}$ \\
Output power & $50 \mathrm{~kW}$ \\
Pulse length & $60 \mathrm{~ns}$ at $1.4-5.6 \mathrm{~km}$ range \\
\hline
\end{tabular}

system, however, represents the echo signal as a three-bit word, thus effectively reducing the image intensity resolution to eight levels. For a radar range setting of $1.4 \mathrm{~km}$ (used for all measurements) each pixel represented an area of $5.3 \times 5.3 \mathrm{~m}$. The digitized images were transferred to a VAX $11 / 750$ computer for analysis. The three-dimensional Fourier analysis of the data utilized 32 consecutive images representing $69 \mathrm{~s}$ of data, from each of which a region of $128 \times 128$ pixels $(679 \times 679 \mathrm{~m})$ was extracted. Such time and space series yield spectral resolution parameters of $\Delta k=9.3 \times 10^{-3} \mathrm{rad} \mathrm{m}^{-1}$ and $\Delta \omega=9.1 \times 10^{-2} \mathrm{rad} \mathrm{s}^{-1}$ with the Nyquist wave number $k_{N}=0.59 \mathrm{rad} \mathrm{m}^{-1}$ and Nyquist frequency $\omega_{N}=1.45 \mathrm{rad} \mathrm{s}^{-1}$.

The full three-dimensional Fourier transform represents a considerable computational effort, involving in excess of 500,000 one-dimensional transforms. Such a series of computations takes approximately 2 hours on the VAX 11/750, the majority of this time being required for disk input/output operations necessitated by the enormous memory requirements for such an analysis.

\section{Wave Spectra Measurements}

The radar system described in section 5 was deployed on board the Deutsches Hydrographisches Institut research vessel Gauss during an experiment near the Island of Sylt $\left(55^{\circ} 00^{\prime} \mathrm{N}\right.$, $7^{\circ} 54^{\prime} \mathrm{E}$ ) on July 4,1982 . In addition to the radar system a pitch-roll-heave buoy and an Aanderra current meter were also deployed. The water depth in the area is $12.5 \mathrm{~m}$ and during the experiment the wind velocity was $9.7 \mathrm{~m} \mathrm{~s}^{-1}$ from a direction of $267^{\circ}$.

The data from the radar was digitized as described in section 5 , and the three-dimensional spectrum $E\left(k_{x}, k_{y}, \omega\right)$ was evaluated. Figure 4 shows a contour plot of this spectrum. Each plane represents the two-dimensional wave number space for a given value of frequency $f=\omega /(2 \pi)$. For comparative purposes the dispersion relationship in still water (equation (9)) has also been included in the figure. The spectrum has been normalized such that the spectral peak has a value of 1.0 and contours have been drawn from 0.9 to 0.1 in increments of 0.2. The minimum contour level is well above the background noise and thus prevents the introduction of spurious values

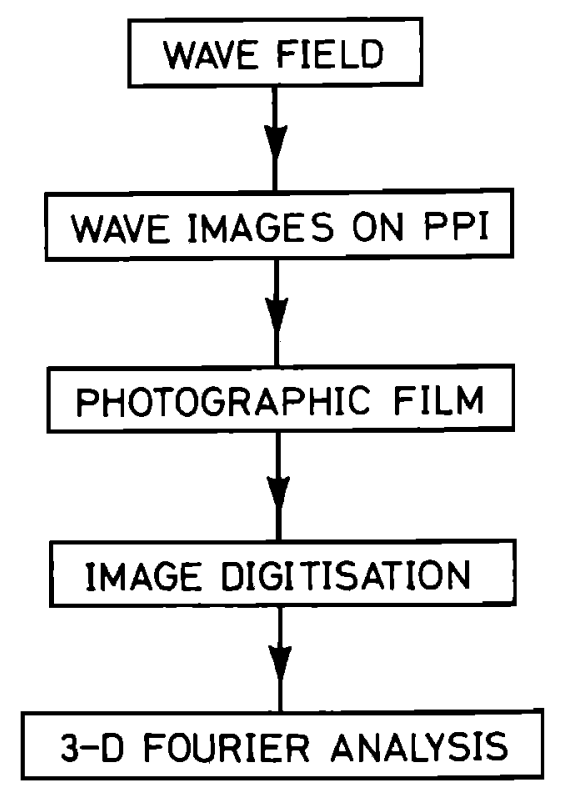

Fig. 3. Flow diagram showing the data recording and analysis procedure. 


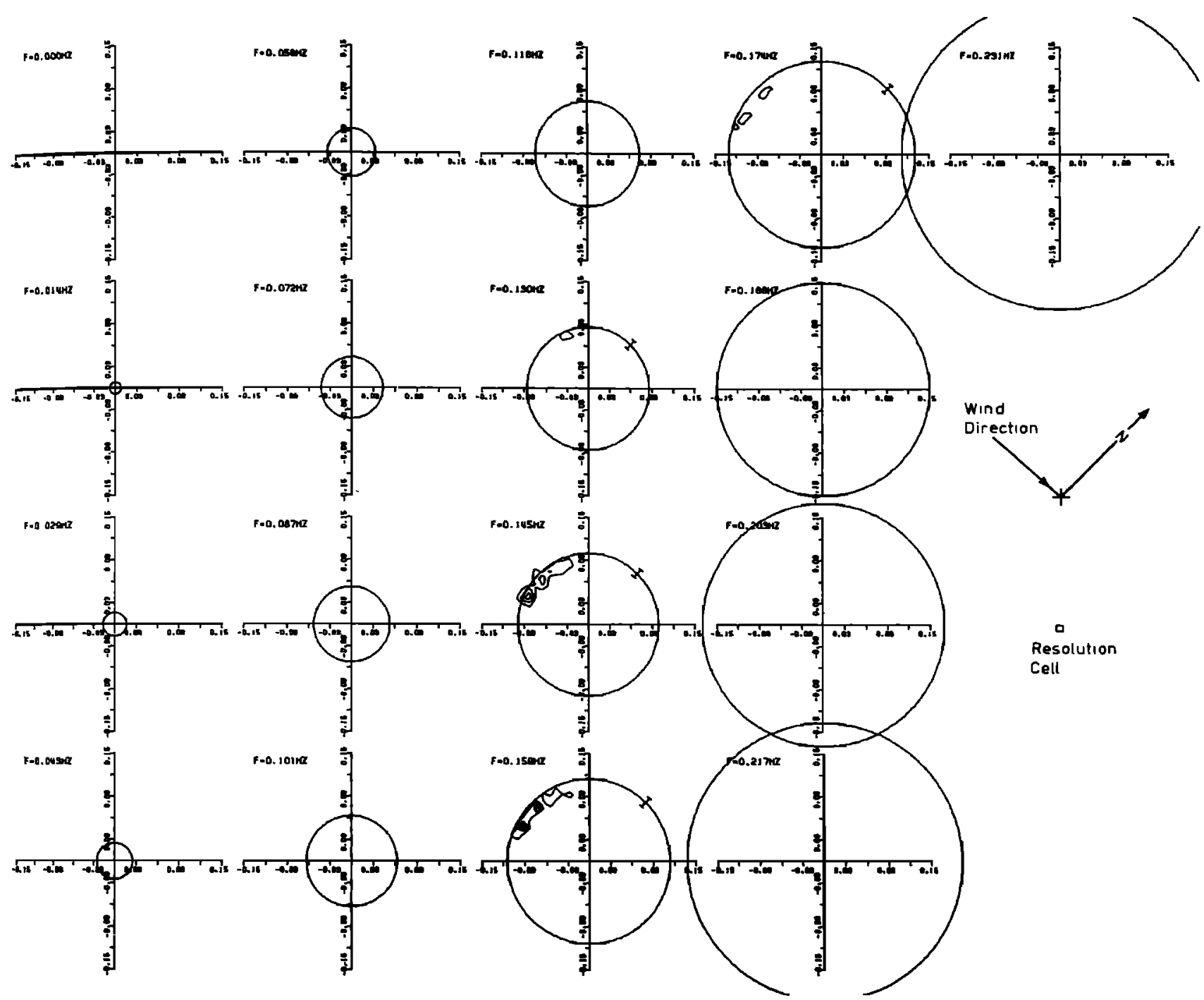

Fig. 4. The three-dimensional $E\left(k_{x}, k_{y}, \omega\right)$ spectrum (from the radar data) with the still water dispersion relationship superimposed. The error bars on the dispersion relationship represent the possible error due to the finite frequency resolution $\Delta \omega$.

into the figure. The spectral energy is concentrated in four frequency planes between 0.130 and $0.174 \mathrm{~Hz}$. In theory the spectral values should be confined to a single line on each plane, defined by the dispersion relationship ((9) for still water or (10) in the presence of a current). The data of Figure 4, however, appears to be smeared over a narrow but finite band. This smearing is purely a result of the contouring routine and the rather coarse wave number resolution. The wave number resolution $\Delta k$ is shown in Figure 4 and is clearly of the same order as the smearing.

Although the three-dimensional spectrum provides considerable information about the wave field, it is more usual to present results in terms of the two dimensional frequency spectrum $E(f, \theta)$ or the one-dimensional $E(f)$ spectrum. The $f$ is given by $\omega /(2 \pi)$ as a function of $\mathbf{k}$ where $\omega$ is calculated from (10) and $\theta$ is the angle of $\mathbf{k}$ in polar coordinates. These alternative spectral representations can be related to the threedimensional spectrum by

$$
\begin{gathered}
E(f, \theta)=|\mathbf{k}| \frac{d|\mathbf{k}|}{d f} \int_{0}^{\infty} E\left(k_{x}, k_{y}, \omega\right) d \omega \\
E(f)=\int_{0}^{2 \pi} E(f, \theta) d \theta
\end{gathered}
$$

The factor in front of the integral in (13) is the Jacobian $\partial\left(k_{x}\right.$, $\left.k_{y}\right) / \partial(f, \theta)$ between the two-coordinate systems $\left(k_{x}, k_{y}\right)$ and $(f$, $\theta)$. Figure 5 shows a comparison between the two-dimensional spectrum obtained from the radar and that obtained by the pitch-roll buoy; the pitch-roll buoy data was analyzed using the technique of Longuet-Higgins et al. [1963]. In both figures the spectra have been noimalized such that the spectral peak has a value of 1.0. The contours have been drawn at an interval of 0.1 to a minimum value of 0.1 . The radar spectrum (Figure 5a) exhibits a bimodal directional distribution with the major peak at $253^{\circ}$ and a smaller but still significant peak at $270^{\circ}$. Both of these peaks have a frequency of approximately $0.15 \mathrm{~Hz}$. Whether this bimodal structure is a true indication of the wave field or a result of the statistical variability of the spectrum is not clear. The directional distribution also appears to be skewed with a more rapid decrease in energy towards the south than the north. This feature is more clearly illustrated in Figure 6, which shows the directional distribution at four selected frequencies. The directional spectrum obtained from the pitch-roll buoy (Figure $5 b$ ) has a spectral peak at $285^{\circ}$ and a frequency of $0.15 \mathrm{~Hz}$. The mean direction of the pitch-roll buoy spectrum is approximately $15^{\circ}$ further to the north than for the radar spectrum. This difference is considered to be quite minor when the possible measurement 


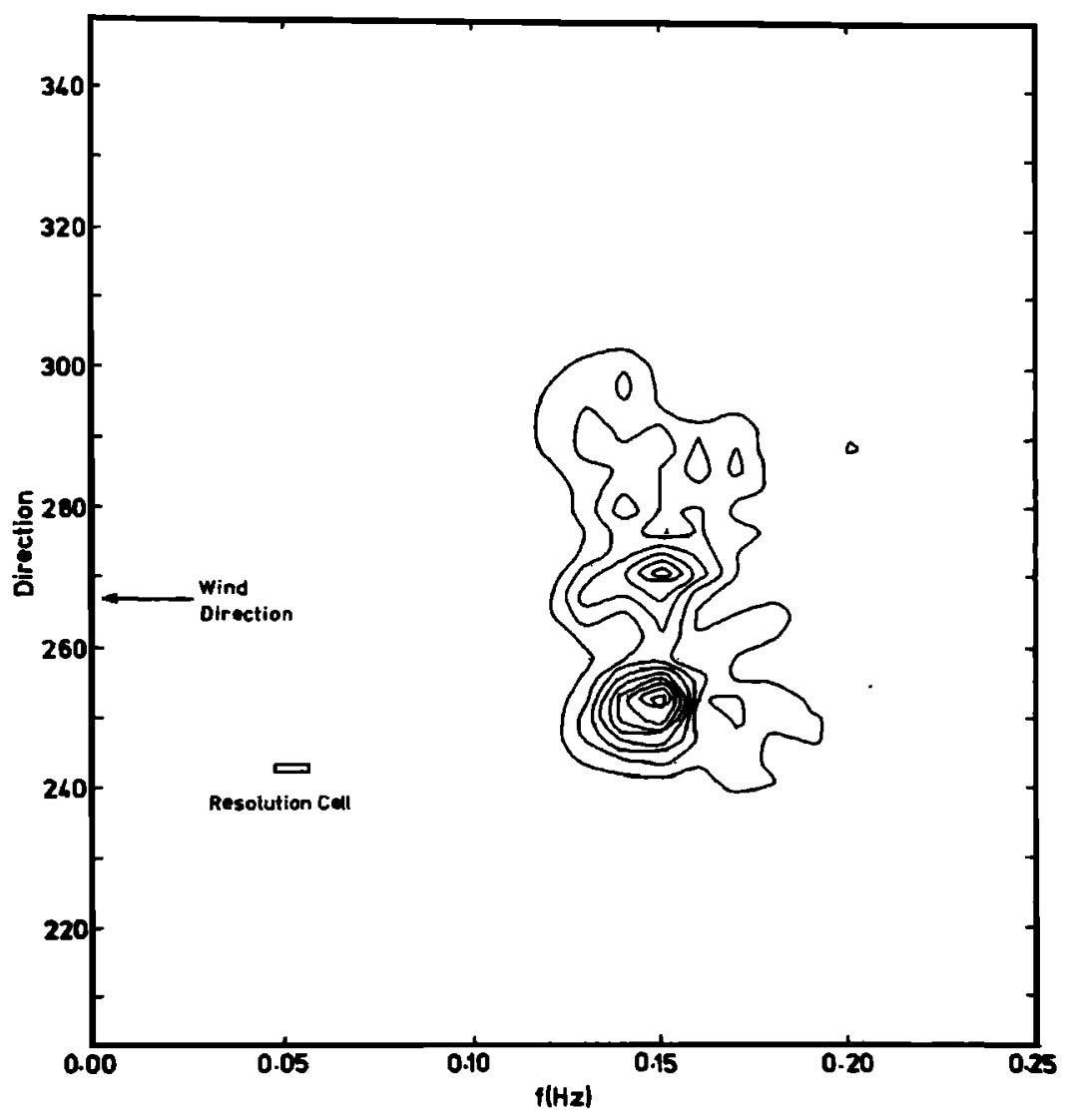

Fig. 5a. The two-dimensional $E(f, \theta)$ spectrum obtained from the radar system.

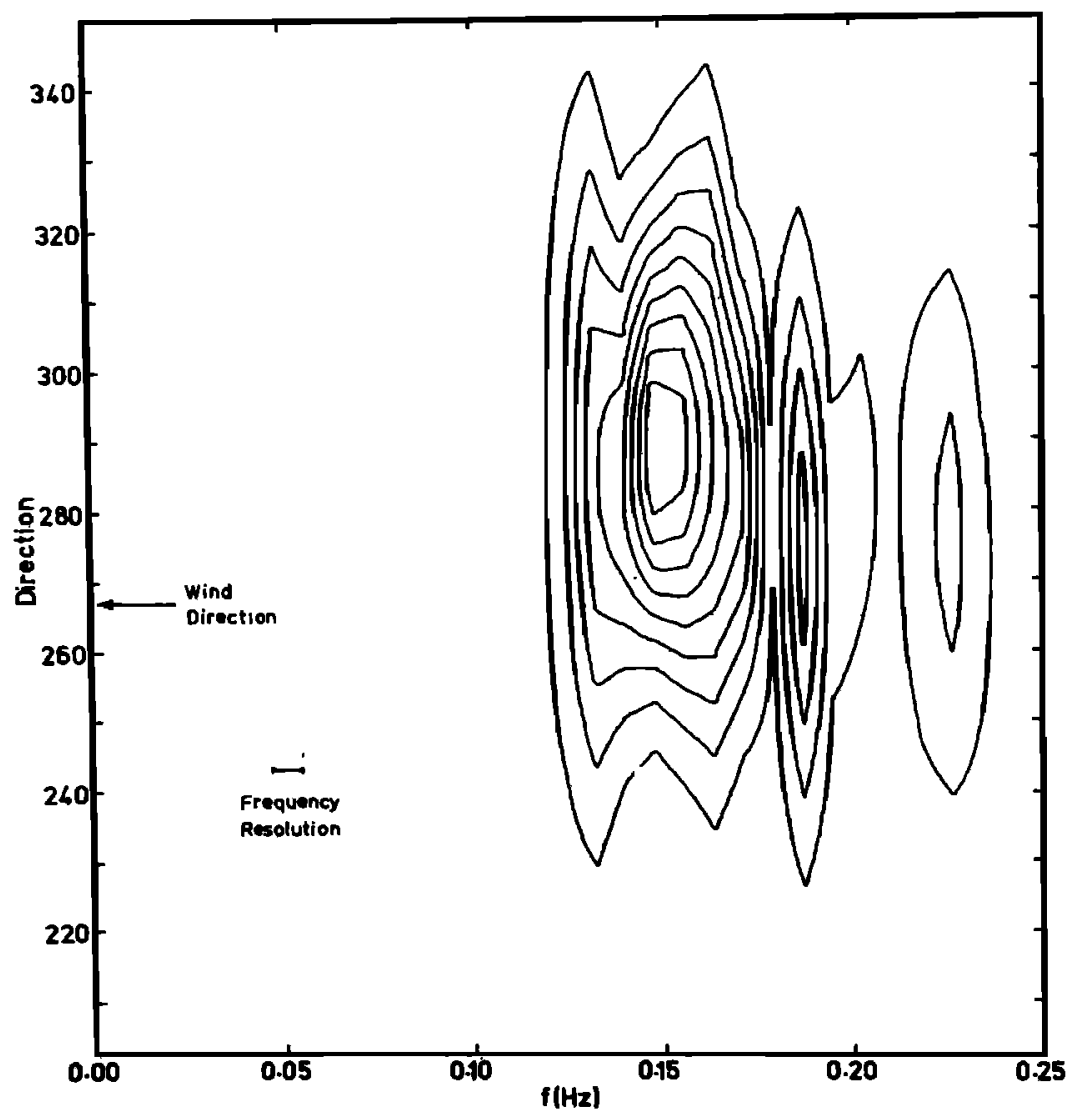

Fig. $5 b$. The two-dimensional $E(f, \theta)$ spectrum obtained from the pitch-roll buoy. 

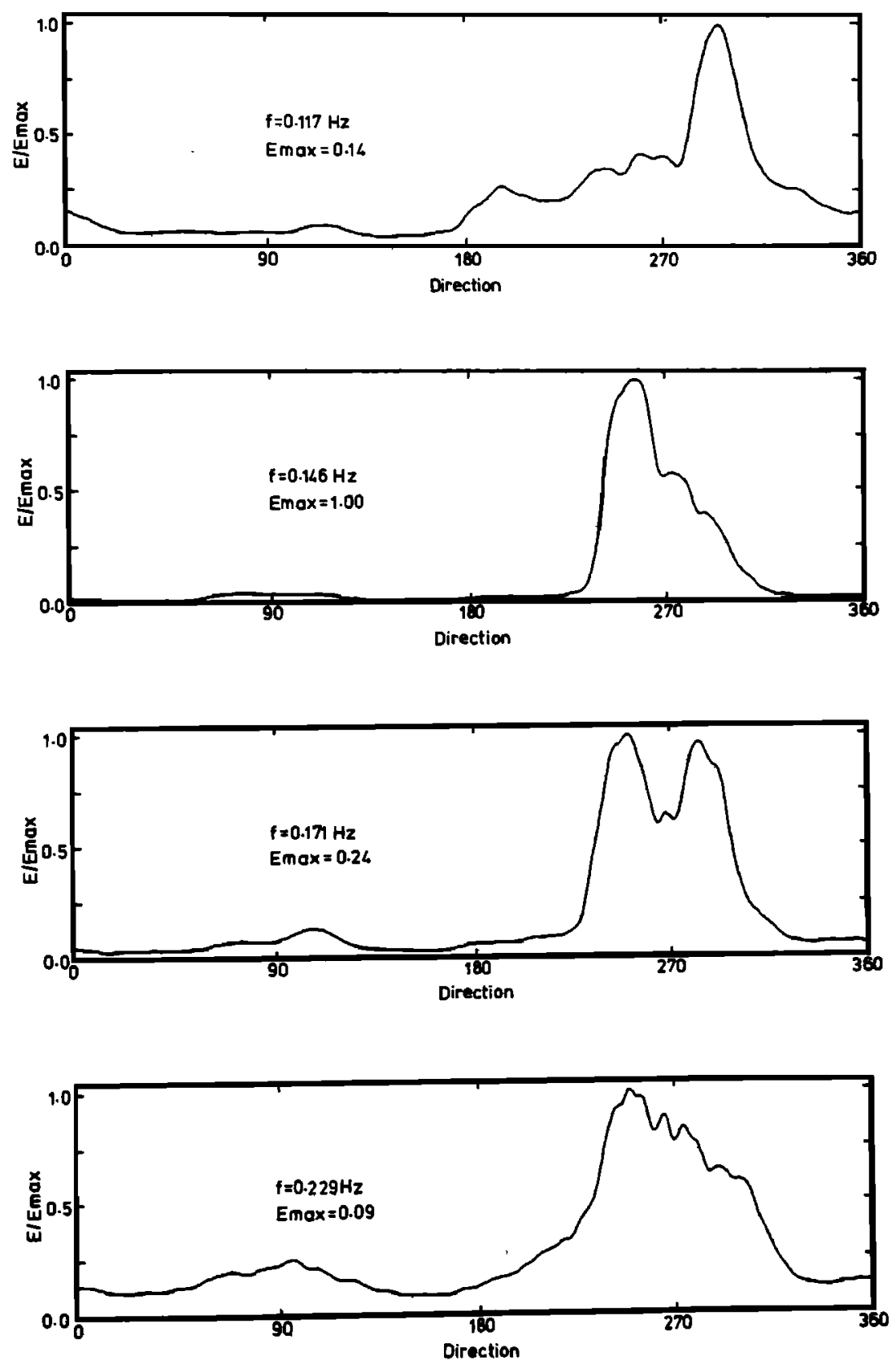

Fig. 6. The directional distribution of the wave energy (from the radar) for four selected frequencies,

errors of both systems are considered. The pitch-roll buoy spectrum also has a broader directional distribution than the radar spectrum. This is possibly due to the dependency of the radar transfer function upon the look diréction of the scanner relative to the wind direction. Ziemer et al. [1983] have shown that the radar return decreases slightly as the angle between the radar view direction and wind direction increases. Hence if the waves are distributed approximately about the wind direction, the radar spectrum would have a narrower directional distribution than the wave spectrum. A more quantitative description of the radar transfer function dependence on the view angle and the distortion of the angular distribution is in progress.

It should be pointed out that although the pitch-roll buoy spectrum provides an estimate of the two-dimensional wave spectrum, it is a very idealized approximation. The pitch-roll buoy analysis relies on the assumption that the waves have a $\cos ^{2 s} \theta / 2$ distribution about the mean direction. Although such a distribution has been widely accepted, it is merely an approximation to the true situation. Such a distribution precludes the existence of bimodal directional distributions as recorded by the radar. Hence in comparing the radar and pitch-roll buoy spectra, it should be remembered that both represent approximations to the wave spectrum. Should it be possible to accurately determine the radar transfer function then the "true" wave spectrum could be determined. Such a potential does not however exist for the pitch-roll buoy measurements. The fact that the directional spectra from the two systems yield compatible results is very encouraging.

Figure 6 indicates that in addition to the energy which is concentrated near $270^{\circ}$ (approximate wind direction), a second and much smaller peak is present at approximately $90^{\circ}$. This peak is quite clearly above the noise level and appears to represent a second wave train propagating in opposition to 


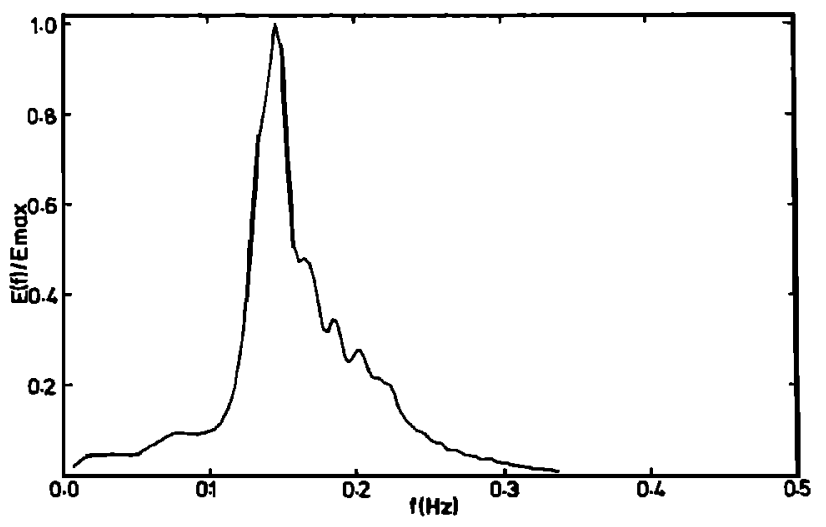

Fig. 7a. The one-dimensional $E(f)$ spectrum obtained from the radar system.

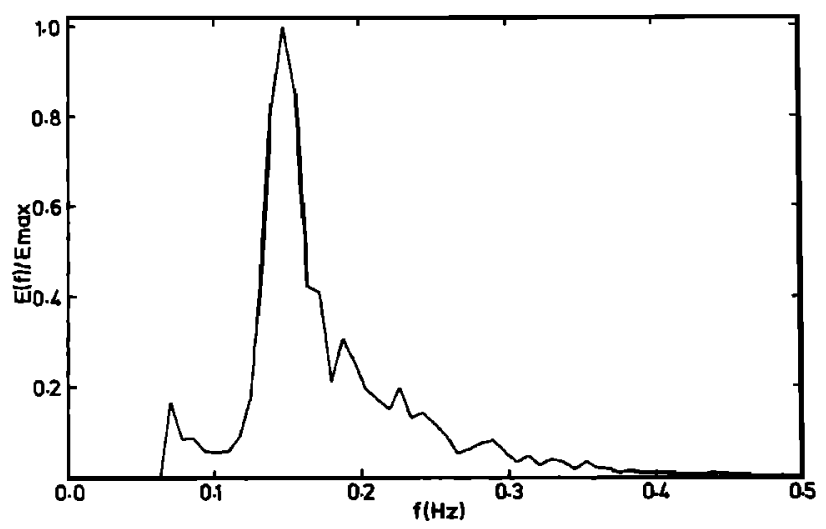

Fig. 7b. The one-dimensional $E(f)$ spectrum obtained from the pitch-roll buoy.

the wind. The ratio of upwind to downwind spectral energy increases with increasing frequency from $4 \%$ at $f=0.15 \mathrm{~Hz}$ to $15 \%$ at $f=0.20 \mathrm{~Hz}$. The existence of such upwind travelling waves can be explained by a number of different mechanisms. These processes include reflections from the shore, wave scattering by the irregular bottom [Long, 1973] and resonant nonlinear interactions within the wave spectrum [Crombie et al., 1978]. The relative importance of the various mechanisms is impossible to determine without a more detailed analysis. Because of the relatively slowly shelving shore in the area, however, it is unlikely that reflections from the shore would be significant.

The presence of this second wave train is a clear indication of the advantages of using the three-dimensional Fourier analysis to remove the directional ambiguity. Had a conventional two-dimensional analysis been used, the upwind travelling waves would have been hidden in the spurious dual image.

The one-dimensional $E(f)$ spectra obtained from the radar and the pitch-roll buoy are also compared in Figure 7. The similarity of the two spectra is clearly apparent; both have a peak at approximately $0.15 \mathrm{~Hz}$ with a steeply rising lowfrequency face and a more slowly falling high-frequency tail. The similarity of the spectra confirms the result of Ziemer et al. [1983] that the transfer function is relatively flat as a function of frequency.

\section{ACCURACy and Statistical Significance}

An advantageous side effect of the three-dimensional Fourier analysis is the very high signal to noise ratio obtained.
Defining the signal to noise ratio $S / N$ as the ratio of the energy at the spectral peak to the background energy level well away from the dispersion shell, values in excess of 1000 are typical for the three-dimensional spectrum. This high value for $S / N$ can be explained if it is assumed that the noise has a "white noise" spectral distribution in space and time. We may consider $E(\Omega)$ in (5) as a white noise spectrum $E_{n}(\Omega)$ with a total variance $\sigma_{n}{ }^{2}$. White noise means that the ensembleaveraged $E_{n}(\Omega)$ is constant in $\Omega$ space. With the integration area in (5) denoted by $V\left(\Omega_{N}\right)$, we deduce

$$
E_{n}(\boldsymbol{\Omega})=\sigma_{n}{ }^{2} / V\left(\Omega_{N}\right)
$$

for the white noise spectral density. We integrate (15) between the Nyquist limits in $k$ space $-\mathbf{k}_{N}, \mathbf{k}_{N}$ and get

$$
\int_{-k_{x N}}^{k_{x N}} d k_{x} \int_{-k_{y N}}^{k_{y, N}} d k_{y} E_{n}(\Omega)=\sigma_{n}{ }^{2} V\left(T_{N}\right)
$$

where $V\left(T_{N}\right)$ is the integration area in $\omega$ space between the Nyquist frequency limits $-\omega_{N}$ and $\omega_{N}$.

In addition, we consider the case where $E(\Omega)=E_{\Omega}(\Omega)$ in (5) results from an ideal wave field (without any noise) with a variance $\sigma_{s}{ }^{2}$ for the wave record. Due to the dispersion relation between $\omega$ and $\mathbf{k}$, the three-dimensional spectrum behaves like a $\delta$ function for the integration variable $\omega$. It vanishes at all $\omega$ values that do not fulfill the dispersion relation. Therefore the integral in (5) can be confined to go over the appropriate resolution cell $\Delta=\Delta\left(k_{x}, k_{y}\right)$ in $\omega$ space only, which is determined by the dispersion relation

$$
\int_{-k_{x N}}^{k_{x N}} d k_{x} \int_{-k_{y N}}^{k_{y N}} d k_{y} \int_{\Delta} d \omega E_{s}\left(k_{x}, k_{y}, \omega\right)=\sigma_{s}{ }^{2}
$$

Since $\Delta$ is given by $1 / T$ we get

$$
\int_{-k_{x N}}^{k_{x N}} d k_{x} \int_{-k_{y N}}^{k_{y N}} d k_{y} E\left[k_{x}, k_{y}, \omega\left(k_{x}, k_{y}\right)\right]=\sigma_{s}^{2} T
$$

$\omega\left(k_{x}, k_{y}\right)$ is given by $(10)$.

The ratio between (18) and (16) shows the increase of signal to noise ratio with length $T$ of the time series.

Spurious effects such as variations in the radar screen intensity or errors due to the alignment of consecutive images in the digitization process will show up as energy well away from the dispersion shell. Since such energy is obviously not associated with the wave field, it can easily be suppressed. An example of such an effect is the variation in the radar screen intensity with distance from the centre. Such energy appears in the three-dimensional analysis at zero frequency and has been suppressed in the spectra presented here.

In interpreting spectral estimates it is necessary to determine their statistical significance. The three-dimensional radar spectrum presented in Figure 4 is the raw spectrum obtained from the data without any averaging. Hence each of the spectral estimates will have a chi squared probability distribution with only two degrees of freedom [Jenkins and Watts, 1968]. Therefore the standard deviation of the spectral estimates will be as large as the true spectral values. Such a large random error would be unacceptable for most applications but is no great hindrance in this situation, as the spectrum has only been used to determine at which wave number frequency veotors there is energy significantly above the background noist. No attempt has been made to determine the actual mas: nitudes of these spectral estimates. The statistical significance of the spectrum could have been improved by averaging over adjacent values in either wave number space or frequency 
space or both. This, however, was not possible because of relatively poor resolution of both wave number and frequency.

Although the three-dimensional spectrum has only two degrees of freedom it can be shown [Jenkins and Watts, 1968] that the integrals in (13) and (14) will insure that $E(f, \theta)$ and $E(f)$ have higher degrees of significance. The integrated spectra are approximately chi squared distributed with $\mu$ degrees of freedom, where

$$
\mu=2\left[\sum_{i=1}^{N} E_{i}\right]^{2}\left[\sum_{i=1}^{N} E_{i}^{2}\right]^{-1}
$$

where the integrals have been replaced with summations over the discrete spectral values. For the current spectra, (15) gives values near the spectral peak of $\mu=3.2$ for $E(f, \theta)$ and $\mu=76.0$ for $E(f)$. Hence the two-dimensional spectrum is still statistically quite variable, whereas the one-dimensional spectrum will be a good estimate of the true spectrum.

In assessing the spectra obtained from the radar system, the spatial resolution of the radar should also be considered. The radar resolution $\delta r$ along a radial line is determined by the pulse length and can be shown to be [Mattie and Harris, 1979]

$$
\delta r=\frac{1}{2} c \tau
$$

where $c$ is the velocity of electromagnetic radiation and $\tau$ is the radar pulse length. For the present system with $\tau=60 \mathrm{~ns}$, $\delta r=9 \mathrm{~m}$. This value could be improved by reducing the pulse length but this would also reduce the power of the returned signal. The angular resolution normal to the radius, $\delta a$, is given by

$$
\delta a=r \delta \theta
$$

where $r$ is the radial distance and $\delta \theta$ is the horizontal beam width of the radar. From Table $1, \delta \theta=0.85^{\circ}$, with (17) indicating that the angular resolution will be equal to the radial resolution at a range of approximately $600 \mathrm{~m}$. Since this figure corresponds approximately to the center of the analyzed images, it is reasonable to assume that the radar resolves a region $9 \times 9 \mathrm{~m}$. For deep water conditions the highest wave frequency which could be measured without aliasing is consequently $0.38 \mathrm{~Hz}$. To obtain an accurate estimate of the spectral density, however, requires a minimum of four sampling points within each wavelength. Thus the accurate highfrequency resolution limit of the radar is $0.21 \mathrm{~Hz}$. It is believed that such resolution is adequate for most oceanographic purposes.

\section{Determination of the Surface Currents}

The three-dimensional spectrum $E\left(k_{x}, k_{y}, \omega\right)$ (Figure 4) shows a consistent deviation from the still water dispersion relationship (9), with the energy lying at lower values of $|\mathbf{k}|$ than would be expected. Even when the possible variation in the dispersion relationship due to the frequency resolution $\Delta \omega$ is considered (see Figure 4), there is still a deviation. The magnitude of this deviation can be more easily seen by firstly determining $E(|\mathbf{k}|, \theta, \omega)=\mathbf{E}\left(k_{x}, k_{y}, \omega\right)|\mathbf{k}|$ and then integrating this spectrum with respect to direction, to give

$$
E(|\mathbf{k}|, \omega)=\int_{0}^{2 \pi} E(|\mathbf{k}|, \theta, \omega) d \theta
$$

This spectrum is presented in Figure 8 together with the dispersion relationship and clearly shows that the data lies at lower values of $|\mathbf{k}|$ than would be expected. As indicated in section 4 , a surface current could be responsible for such a deviation.

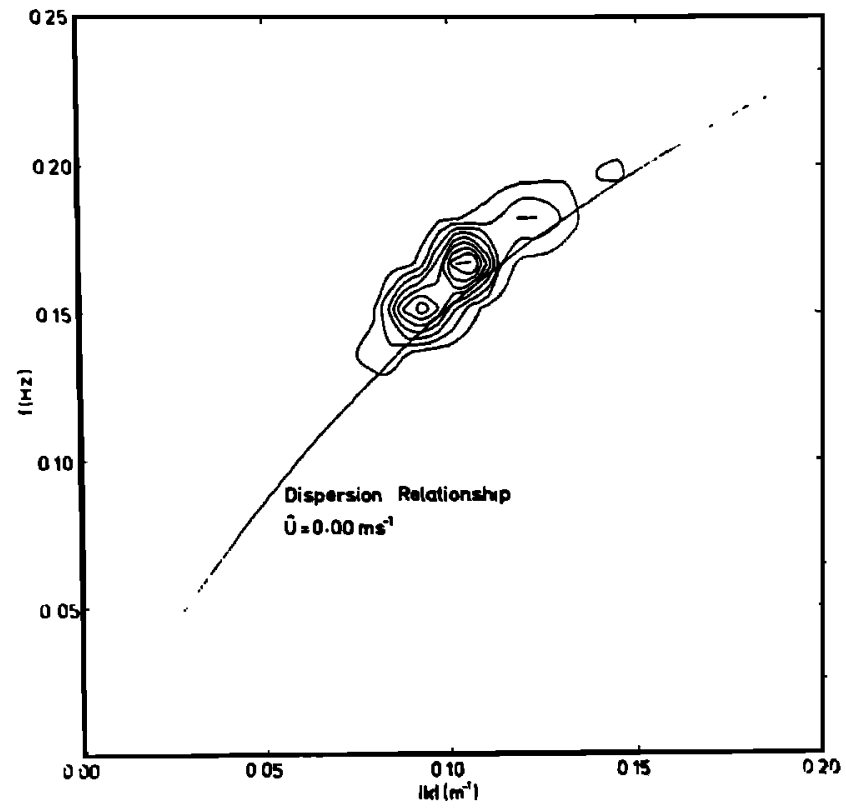

Fig. 8. The integrated two-dimensional $E(|\mathbf{k}|, \omega)$ spectrum (from the radar) with the still water dispersion relationship superimposed.

In an effort to determine whether a current is responsible for this deviation, a least squares curve fitting technique was used to fit a curve of the form $\omega=\omega_{0}+\bar{U}_{x} k_{x}+\bar{U}_{y} k_{y}$ to the data. The terms $\tilde{U}_{x}$ and $\tilde{U}_{y}$ are the best fit components in the $x$ (east) and $y$ (north) directions, respectively, of the effective current (equation (10)) sensed by the waves. This least squares analysis yielded values of $\tilde{U}_{x}=0.49 \mathrm{~m} \mathrm{~s}^{-1}$ and $\tilde{U}_{y}=-0.41 \mathrm{~m}$ $\mathrm{s}^{-1}$, indicating a current of magnitude $|\tilde{\mathrm{U}}|=0.64 \mathrm{~m} \mathrm{~s}^{-1}$ from $310^{\circ}$. An indication of how well the least squares approximation fits the data can be obtained from the normalized variance of the curve fit, $V$, where $V$ is defined as

$$
\begin{aligned}
V=\left\{\sum_{i} \sum_{j}\left[\omega\left(k_{x i}, k_{y j}\right)-\tilde{\omega}\left(k_{x i}, k_{y j}\right)\right]^{2}\right\} \\
\cdot\left\{\sum_{i} \sum_{j}\left[\omega\left(k_{x i}, k_{y j}\right]^{2}\right\}^{-1}\right.
\end{aligned}
$$

$\omega\left(k_{x i}, k_{y j}\right)$ is the frequency at which spectral energy is located for wave number components $k_{x i}, k_{y j}$ and $\tilde{\omega}\left(k_{x i}, k_{y j}\right)$ is the corresponding value from the least squares model. $A$ value of $V=0$ indicates that the least squares model fits the data perfectly. The present data yields $V=1.3 \times 10^{-3}$, indicating that the modified dispersion relationship is a very good approximation to the data. The three-dimensional spectrum $E\left(k_{x}, k_{y}\right.$, $\omega)$ and the integrated $E(|k|, \omega)$ spectrum, together with the modified dispersion relationship, are shown in Figures 9 and 10 , respectively. Both of these figures clearly illustrate how well the least squares model agrees with the data. Such good agreement is a strong indication that the deviation from the still water dispersion relationship is due to the presence of a surface current.

During the experiment an Aanderra current meter was deployed which indicated a current of $0.45 \mathrm{~m} \mathrm{~s}^{-1}$ from $350^{\circ}$. This result cannot, however, be directly compared with the current measured by the radar. As shown by (11), the radar measures a depth integrated current which is probably nearer the surface current than that measured by the current meter, which was deployed at a depth of $10.5 \mathrm{~m}$. An order of magnitude estimate of the surface current can be obtained by considering the combined effects of the tidal current together with 


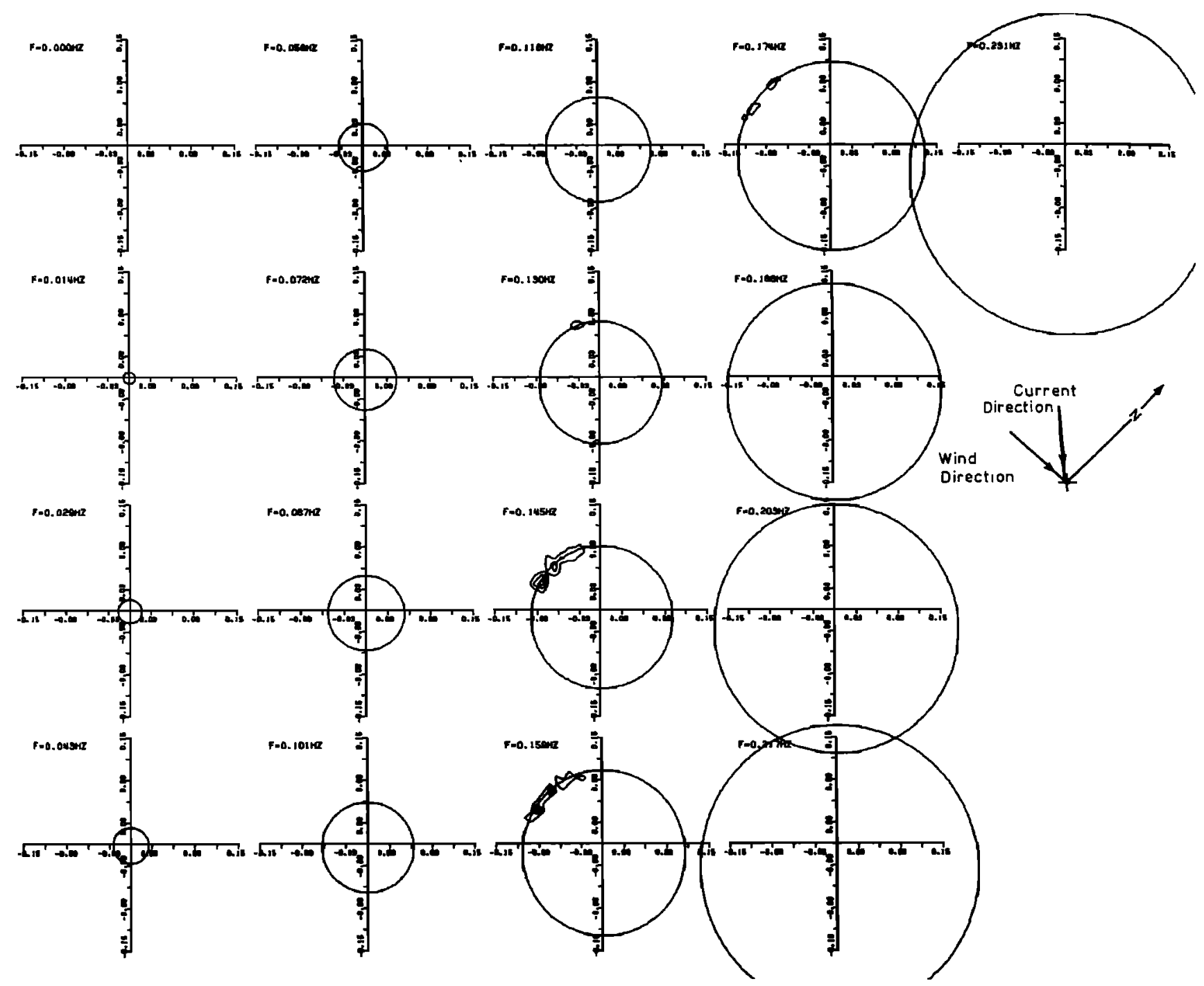

Fig. 9. The three-dimensional $E\left(k_{x}, k_{y}, \omega\right)$ spectrum (from the radar) with the Doppler shifted dispersion relationship superimposed.

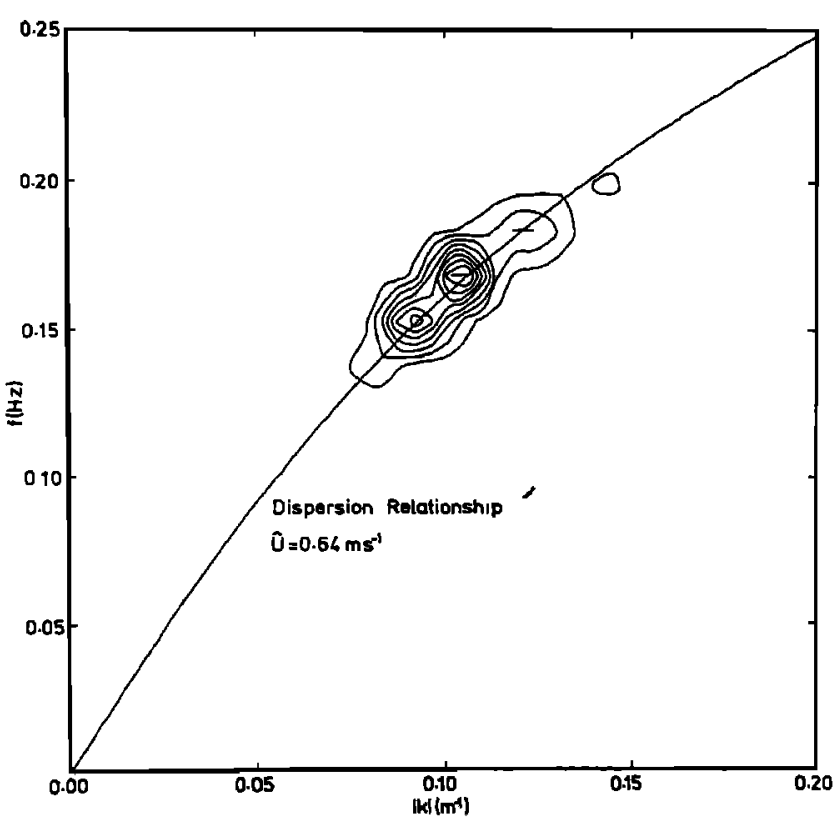

Fig. 10. The integrated two-dimensional $E(|\mathbf{k}|, \omega)$ spectrum (from the radar) with the Doppler shifted dispersion relationship superimposed. the wind drift current. Tide tables for the German Bight indicate a tidal flow of $0.36 \mathrm{~m} \mathrm{~s}^{-1}$ from $340^{\circ}$. As stated earlier the wind velocity was $9.7 \mathrm{~m} \mathrm{~s}^{-1}$ from $267^{\circ} . W u$ [1975] has found that the surface drift current is approximately $3.5 \%$ of the wind velocity. This gives a surface drift of $0.34 \mathrm{~m} \mathrm{~s}^{-1}$ and, allowing for the Ekman effect [Ekman, 1905] the approximate direction is from $315^{\circ}$. The vector sum of these currents yields a value of $0.69 \mathrm{~m} \mathrm{~s}^{-1}$ from $326^{\circ}$. This value is in surprisingly good agreement with the radar inferred value of $0.64 \mathrm{~m} \mathrm{~s}^{-1}$ from $310^{\circ}$. Such a comparison is far from conclusive but indicates that the radar determined current is quite plausible.

\section{ConClusions}

That conventional marine radars return images from ocean waves has been known for some time, although the potential of such images has received scant attention. We have shown that a three-dimensional Fourier analysis of such images yields valuable information on both the surface wave field and near surface currents. The introduction of the third time dimension into the analysis has the advantages of providing information on the wave phase speed and hence the mas nitude and direction of near surface currents, removing the directional ambiguity inherent in the two-dimensional analysis, and providing an extremely high signal to noise ratio. The spectra and surface currents determined by the radar system 
are in good agreement with the available ground truth da ta indicating that such radar systems represent a reliable a nd very cost-effective means of measuring a number of perrinent oceanographic parameters.

Acknowledgments. This study was conducted within the SODderforschungsbereich 94 "Meeresforschung" of the Univer sity of Hamburg with financial support from the Deutsche Forsche angs ge meinschaft. We gratefully acknowledge the assistance of $\mathbf{R}$. Dorf $\vec{P}_{\text {er }}$ from G.K.S.S. Geesthacht in digitization of the photographs. One of us (I. R. Young) is grateful to K. Hasselmann and J. Sünd ermzann for their hospitality.

\section{ReFERENCES}

Alpers, W., Imaging ocean waves by synthetic aperture ra-dar: A review, in Satellite Microwave Sensing, edited by T. D . All an pp. 107-120, Ellis Horwood, Chichester, U. K., 1983.

Alpers, W., J. Schröter, F. Schlude, H. J. Müller, and K. P. KKolt-ermann, Ocean surface current measurements by an $L-b a n-d i n$. frequency microwave scatterometer, Radio Sci, , 16(1), 93-100 - 1981.

Atanassov, V., W. Rosenthal, and F. Ziemer, Removal of ambiguity of two-dimensional power spectra obtained by processing ship nälar images of ocean waves, J. Geophys. Res., this issue.

Barrick, D. E., M. W. Evans, and B. L. Weber, Ocean surface cemrents mapped by radar, Science, 198, 138-144, 1977.

Bath, M., Spectral Analysis in Geophysics, 563 pp,- Elsevie, N N York, 1974.

Brekhovskikh, L. M., Waves in Layered Media, 460 pp., Acenderneic New York, 1960.

Crombie, D. D., K. Hasselmann, and W. Sell, High-frequency nadar observations of sea waves travelling in opposition to the pirad, Boundary Layer Meteorol., 13, 45-54, 1978.

Ekman, V. W., On the influence of the earth's rotation on oce-an currents, Arch. Math. Astron. Phys., 2(11), 1905.

Evmenov, V. F., et al., Test of the radar method of defiming ocezan waves elements, Fluid Mech. Sov. Res., 2(5), 141-145, 1973.

Hoogeboom, P., and W. Rosenthal, Directional wave spect ra irs rad 4 ar images, paper presented at the International Geoscience and Remote Sensing Symposium, IEEE Geosci. and Remote Sensian Soc, Munich, June 1-4, 1982.

Ijima, T., T. Takahashi, and H. Sasaki, Application of rgdars to pa . ve observations, Proc. Conf. Coastal Eng. I lth, 30(1), 10-22, 1964.

Jenkins, G. M., and D. G. Watts, Spectral Analysis ane its app-li. cations, 525 p., Holden-Day, San Francisco, 1968.

Kolmykov, A. I., and V. V. Pustovoytenko, On polarization featur-e of radio signals scattered from the sea surface at small ravi-as angles, J. Geophys. Res., 81, 1960-1964, 1976.
Leang, R. B., Scattering of surface waves by an irregular bottom, $J$. Geophys. Res., 78, 7861-7970, 1973.

Loomu-et-Higgins, M. S., and O. M. Phillips, Phase velocity effects in teti=ary wave interactions, J. Fluid Mech., I2, 333-336, 1962

Longuet-Higgins, M. S., D. E., Cartwright, and N. D. Smith, Observatins so the directional spectrum of sea waves using the motions of a to ating buoy, in Ocean Wave Spectra, pp. 111-132, Prentice-Hall, Engluewood Cliffs, N. J., 1963.

W It Itie-, M. G., and D. L. Harris, A system for using radar to record whe direction, Tech. Rep. 79-1, 50 pp., U.S. Army Corps of Eng. Cos St. Eng. Res. Ctr., Fort Belvoir, Va., 1979.

M.Idkish, W., and D. B. Ross, Imaging radar observations of directhel properties of ocean waves, J. Geophys. Res., 88, 4407-4419, 1983 :.

Qu ulsh-oorn, H. M., The use of radar in hydrodynamic surveying, Proe -. Conf. Coastal Eng. 7th, 59-76, 1960.

Phtrillps, O. M., The Dynamics of the Upper Ocean, 2nd ed., $336 \mathrm{pp}$. - anubridge University Press, London, 1977.

SeI $=$ lber - J. E., Spatial densities for specular points on a Gaussian : Surfece, IEEE Trans. Antennas Propag., AP-20, 723-731, 1972

Snrrith, B. G., Geometrical shadowing of a random rough surface, - IRE EE Trans. Antennas. Propag., AP-15, 668-671, 1967.

Stement, R. H., and J. W. Joy, HF radio measurements of surface - clurents, Deep Sea Res., 2l, 1039-1049, 1974.

Parelarmula G. R., Theories for the interaction of electromagnetic and -

Hs age=r, R. T., Shadowing of randomly rough surfaces, J. Acoust. Soc. - Ath, 4l, 138-147, 1967.

WE-alborn-Watt, R., The Use of Radar at Sea, 332 pp., Hollis and - Carteer, London, 1978.

WE- illis ${ }^{-}$T. G., and H. Beaumont, Wave direction measurements using \& sas-urveillance radars, Tech. Memo. TR 118, R. Aircraft Establish- ment, Farnbough, Great Britain, 1971.

Wre righ , F. F, Wave observations by shipboard radar, Ocean Sci. - Oera :nEng. $1,506-514,1965$.

Hu u J, Wind-induced drift currents, J. Fluid Mech., 68, 49-70, 1975.

lieverer F., W. Rosenthal, and H. Carlson, Measurements of direc- tinaal wave spectra by ship radar, paper presented at the IAPSO - Sym posium, General Assembly, Int. Assoc. for Phys. Sci. of the - Oaranns, Hamburg, Federal Republic of Germany, 1983.

-W Rosenthal and I. R. Young, Institut für Meereskunde der Unmivesität Hamburg and Max-Planck-Institut für Meteorologie, th ande-strasse 55-GEOMATIKUM, D-2000 Hamburg 13, Federal let=pobl=ic of Germany.

I F Ziiemer, Institut für Meereskunde der Universität Hamburg and Destros Hydrographisches Institut, D-2000 Hamburg 60, Federal leTspotl=ic of Germany.

(Received March 27, 1984; accepted May 17, 1984.) 